\title{
(2) OPEN ACCESS \\ Serratus anterior plane versus paravertebral nerve blocks for postoperative analgesia after non- mastectomy breast surgery: a randomized controlled non-inferiority trial
}

\author{
Rodney A Gabriel, ${ }^{1,2}$ Matthew W Swisher, ${ }^{1}$ Jacklynn F Sztain, ${ }^{1}$ Brian P Curran, ${ }^{1}$ \\ Engy T Said (1) , 'Wendy B Abramson, ${ }^{1}$ Bahareh Khatibi, ${ }^{1}$ Brenton S Alexander (ㄷ ,' \\ John J Finneran, ${ }^{1}$ Anne M Wallace, ${ }^{3}$ Ava Armani, ${ }^{3}$ Sarah Blair, ${ }^{3}$ Marek Dobke, ${ }^{3}$ \\ Ahmed Suliman, ${ }^{3}$ Christopher Reid, ${ }^{3}$ Michael C Donohue, ${ }^{4}$ Brian M Ilfeld ${ }^{1}$
}

- Additional online supplemental material is published online only. To view, please visit the journal online (http://dx.doi.org/10.1136/ rapm-2021-102785).

'Department of Anesthesiology, Division of Regional Anesthesia, University of California San Diego, La Jolla, California, USA ${ }^{2}$ Department of Medicine, Division of Biomedical Informatics, University of California San Diego, La Jolla, California, USA

${ }^{3}$ Department of Surgery, University of California San Diego, La Jolla, California, USA ${ }^{4}$ Department of Neurology, University of Southern California, Los Angeles, California, USA

Correspondence to Dr Rodney A Gabriel, Department of Anesthesiology, University of California San Diego, La Jolla, USA ragabriel@health.ucsd.edu

Received 12 April 2021 Accepted 28 May 2021 Published Online First 22 June 2021
Check for updates

(c) Author(s) (or their employer(s)) 2021. Re-use permitted under CC BY-NC. No commercial re-use. See rights and permissions. Published by BMJ.

To cite: Gabriel RA, Swisher MW, Sztain JF, et al. Reg Anesth Pain Med 2021:46:773-778

\section{ABSTRACT}

Background Paravertebral and serratus plane blocks are both used to treat pain following breast surgery. However, it remains unknown if the newer serratus block provides comparable analgesia to the decades-old paravertebral technique.

Methods Subjects undergoing unilateral or bilateral non-mastectomy breast surgery were randomized to a single-injection serratus or paravertebral block in a subject-masked fashion (ropivacaine $0.5 \% ; 20 \mathrm{~mL}$ unilateral; $16 \mathrm{~mL} / \mathrm{side}$ bilateral). We hypothesized that (1) analgesia would be non-inferior in the recovery room with serratus blocks (measurement: Numeric Rating Scale), and (2) opioid consumption would be non-inferior with serratus blocks in the operating and recovery rooms. In order to claim that serratus blocks are non-inferior to paravertebral blocks, both hypotheses must be at least non-inferior.

Results Within the recovery room, pain scores for participants with serratus blocks $(n=49)$ had a median (IQR) of $4.0(0-5.5)$ vs $0(0-3.0)$ for those with paravertebral blocks $(n=51): 0.95 \% \mathrm{Cl}-3.00$ to $-0.00 ; p=0.001$. However, the difference in morphine equivalents did not reach statistical significance for superiority with the serratus group consuming $14 \mathrm{mg}$ (10-19) vs $10 \mathrm{mg}(10-16)$ for the paravertebral group: $95 \% \mathrm{Cl}-4.50$ to $0.00, p=0.123$. Since the $95 \% \mathrm{Cl}$ lower limit of -4.5 was less than our prespecified margin of -2.0 , we failed to conclude non-inferiority of the serratus block with regard to opioid consumption. Conclusions Serratus blocks provided inferior analgesia compared with paravertebral blocks. Without a dramatic improvement in safety profile for serratus blocks, it appears that paravertebral blocks are superior to serratus blocks for postoperative analgesia after non-mastectomy breast surgery.

Trial registration number NCT03860974.

\section{INTRODUCTION}

Breast surgery is commonly associated with moderate-to-severe pain and opioid use during the acute postoperative period. ${ }^{1}$ Furthermore, inadequate analgesia during this time is associated with development of persistent postoperative pain. ${ }^{2-4}$
Multimodal analgesia is an important component of surgical management of these patients. ${ }^{5}$ Regional anesthesia is frequently a major factor in multimodal analgesia pathways, and thus, it is essential that such interventions are evaluated and implemented.

Paravertebral nerve blocks (PVBs) have been used to treat postoperative pain following breast surgery for decades. ${ }^{67}$ However, these blocks do have distinct disadvantages such as the risks of hemodynamic instability, neuraxial hematoma, and pleural injury ${ }^{6}$; although major adverse events are reported to be rare. ${ }^{8}$ More recently, fascial plane blocks have been introduced as an alternative to PVBs-these include the erector spinae plane block, ${ }^{9}$ PECS-2 block, ${ }^{10}$ and serratus anterior plane block. $^{11}$

Serratus blocks were introduced by Blanco et $a l^{11}$ and involves deposition of local anesthetic either deep or superficial to the serratus anterior muscle at the level of the fifth rib. It is theorized to provide nerve blockade of the lateral cutaneous branches of the intercostal nerves. ${ }^{12}$ While still possible, ${ }^{13}$ the risk of pneumothorax is theoretically decreased compared with PVBs because of the relatively more superficial placement of this block. Furthermore, the target for local deposition is superficial to osseus structures (ie, ribs) rather than being directly superficial to the pleura. Despite the proliferation of publications describing serratus blocks, prospective, randomized controlled studies comparing serratus blocks to PVB for breast surgery are sparse. ${ }^{14-17}$ It remains unknown if the newer serratus block provides comparable analgesia to the decades-old PVB approach.

We; therefore, performed a randomized, subject-masked, parallel-arm, active-controlled study comparing PVB to serratus block for non-mastectomy breast surgery patients. We hypothesized that serratus blocks would provide non-inferior analgesia to PVBs following breast surgery; with dual primary end points of (1) pain scores within the recovery room and (2) opioid consumption in both the operating and recovery room. In order to claim that serratus blocks are non-inferior to PVBs, both primary endpoints had to be at least non-inferior. 


\section{METHODS}

\section{Study participants}

Written, informed consent was obtained from all participating subjects.

\section{Inclusion and exclusion criteria}

This was a single institution trial. Adults (equal to or greater than 18 years) presenting for unilateral or bilateral breast surgery with at least moderate postoperative pain anticipated and a planned single-injection regional analgesic were screened for enrolment preoperatively. This included lumpectomy with axillary node biopsy/resection, breast reconstruction, breast reduction, mastopexy, and implant expander removal/placement. Exclusion criteria included: simple lumpectomy (with no other procedure), planned regional analgesic with perineural catheter placement, morbid obesity as defined as a body mass index $>40$ $\mathrm{kg} / \mathrm{m}^{2}$, renal insufficiency (preoperative creatinine $>1.5 \mathrm{mg} /$ $\mathrm{dL}$ ), current chronic opioid use (daily equivalent of $>30 \mathrm{mg}$ of morphine within the 2 weeks prior to surgery and duration of use $>4$ weeks), history of opioid abuse, any comorbidity that results in moderate or severe functional limitation, inability to communicate with the investigators or hospital staff, pregnancy, incarceration and allergy to study medications (ropivacaine). Patients undergoing mastectomy were excluded since they are offered a continuous PVB at our institution as standard care.

\section{Block procedures}

Preoperatively, participating subjects were placed in the sitting or lateral decubitus position with standard American Society of Anesthesiologists monitors and supplemental oxygen. All block procedures were performed by a regional anesthesia attending or fellow. Sedation was provided with intravenous midazolam and fentanyl, titrated to patient comfort during the block procedure. For subjects receiving PVB, a low-frequency curvilinear ultrasound transducer $(6-2 \mathrm{MHz})$ was used to identify the T1 through T5 transverse processes and paravertebral spaces. For subjects who received a serratus block, a linear ultrasound transducer $(13-6 \mathrm{MHz})$ was placed at the mid-axillary line to identify the short-axis view of ribs 4 and 5 deep to the serratus anterior muscle. The needle was inserted caudad to the probe.

After confirmation of acceptable ultrasound visualization of both potential block sites, subjects were randomized using a computer-generated list and opaque, sealed envelopes to one of two treatment groups stratified for unilateral versus bilateral surgery: (1) serratus block or (2) PVB. Subjects were blinded to treatment group allocation.

PVB: A 20-gage Tuohy needle was inserted into the appropriate plane/space under direct ultrasound guidance via an in-plane parasagittal approach. Local anesthetic $(0.5 \%$ ropivacaine) with 1:400 000 of epinephrine was injected at the appropriate level(s) after negative aspiration $(20 \mathrm{~mL}$ for unilateral surgery, $16 \mathrm{~mL}$ on each side for bilateral surgery). For PVBs, a two-level injection technique was performed: T2 and T4 for surgeries involving axillary work or T3 and T5 for surgeries not involving axillary work.

Serratus anterior plane block. The subject is placed in a lateral position with operative side up. Ultrasound guidance was placed in a coronal plane at the midaxillary line and rib 4, rib 5, latissimus dorsi, and serratus anterior muscle are identified. A 20-gage Tuohy needle was then inserted in-plane and directed towards rib 5. The needle was inserted caudad to the probe. Local anesthetic ( $0.5 \%$ ropivacaine) with $1: 400000$ of epinephrine was deposited deep to the serratus anterior muscle (20 mL for unilateral surgery, $16 \mathrm{~mL}$ on each side for bilateral surgery). ${ }^{18}$

Blocks were considered successful if, within $30 \mathrm{~min}$, the subject experienced decreased sensation to cold temperature over the level of the ipsilateral fourth thoracic dermatome at the level of anterior axillary line. For subjects undergoing a bilateral surgical procedure, a block using the same protocol was administered on the contralateral side. In addition, preoperatively, patients were given oral acetaminophen. A standardized multimodal analgesic regimen postoperatively was not used and left to the surgical team's discretion.

\section{Intraoperative and postoperative management}

Prior to surgery, all patients received oral $975 \mathrm{mg}$ acetaminophen. Surgery was performed under general anesthesia with a combination of inhaled and intravenous anesthetics. Intraoperative fentanyl was administered at the discretion of the blinded anesthesia team based on cardiovascular responsiveness to noxious stimuli, and total fentanyl use was recorded. Intravenous ketorolac $(0.5 \mathrm{mg} / \mathrm{kg}$, up to $30 \mathrm{mg})$ was also provided at the discretion of the blinded anesthesia team. Surgeons did not inject additional local anesthetic intraoperatively. Subjects were extubated, taken to the postanesthesia care unit (PACU) and received by a nurse blinded to randomization. A standard PACU opioid algorithm was used which involved: (1) intravenous fentanyl 25 $\mu \mathrm{g}$ for Numeric Rating Scale (NRS) pain scores of either 4 or 5 , (2) intravenous fentanyl $50 \mu \mathrm{g}$ for NRS of 5 or greater, (3) intravenous hydromorphone $0.5 \mathrm{mg}$ for NRS of 5 or greater if fentanyl deemed ineffective, and (4) oxycodone $5 \mathrm{mg}$ for NRS of 4-6 if able to tolerate oral medications. Subjects scheduled for outpatient surgery were discharged home with a prescription for oxycodone tablets $(5 \mathrm{mg})$ for supplementary analgesia and instructed to record the time at which they took their first opioid tablet as well as the time at which they believed the block began to resolve. Admitted subjects were provided oxycodone 5 mg every 4 hours as needed for NRS of 4-6; and 10 mg every 4 hours as needed for NRS of 7-10. Reasons for same-day hospital admission includes intractable postoperative nausea/vomiting, severe pain uncontrollable with repeated intravenous opioids, postoperative respiratory depression requiring overnight monitoring, surgical indications (ie, bleeding, preference for overnight monitoring due to surgical reasons). No patients who were planned for same-day discharge were admitted to the hospital for pain, nausea/vomiting, or other complications related to anesthesia.

\section{Outcome measurements}

Pain scores were recorded using the 11 -point NRS $(0-10,0=$ no pain, $10=$ worst pain imaginable). Opioid consumption was analyzed as intravenous morphine equivalents (MEQ). Intraoperative and postoperative intravenous fentanyl, intravenous hydromorphone, and oral oxycodone were converted to intravenous MEQ. Since most of these surgeries were performed on an outpatient basis, we chose to limit our blinded pain assessments to the PACU. We recorded times to perform nerve blocks and procedure-related pain scores. Within the PACU, pain scores, opioid requirements, and antiemetic administration were recorded by nursing staff blinded to treatment. The morning following surgery, all subjects were contacted by telephone or in person (if hospitalized) to record lowest, average, highest, and current pain scores, number of sleep disturbances, and nausea using a $0-10$ Likert scale $(0=$ no nausea; $10=$ vomiting). 
For ambulatory subjects, opioid requirements were recorded by patients while inpatient subjects had opioid requirements extracted from the electronic medical record. In addition, for inpatient subjects, antiemetic use and nursing-recorded pain scores were extracted from the electronic medical record. We collected the times at which subjects identified block resolution and the time at which they consumed their first oral opioid analgesic following PACU discharge. In addition, possible blockrelated side effects and adverse events were recorded.

\section{Statistical analysis}

We hypothesized that (1) analgesia would be non-inferior in the recovery room as measured on an NRS $(0-10)$ with serratus blocks and (2) opioid consumption would be non-inferior in the operating and recovery rooms with serratus blocks. In order to claim that serratus blocks are non-inferior to PVBs, both hypotheses had to be at least non-inferior. We tested the non-inferiority of serratus block compared with PVB using the 95\% CI associated with the Wilcoxon-Mann-Whitney test with continuity correction. If the lower limit of the $95 \%$ CI for median 'average' PACU pain scores was greater than -1.25 (based on PVB minus serratus blocks), we would conclude non-inferiority. The noninferiority of serratus blocks with regard to opioid consumption was similarly tested by comparing the limits of a $95 \%$ CI associated with the Wilcoxon-Mann-Whitney test with continuity correction to a predefined non-inferiority margin of $2 \mathrm{mg}$ intravenous MEQ. Of note, testing for non-inferiority by comparing the limits of conventional two-sided 95\% CIs to prespecified non-inferiority margins does not preclude the possibility of concluding inferiority or superiority if the same CI excludes zero. ${ }^{19}$ All subjects were analyzed on an intention-to-treat basis. R V.3.5.2 (https://www.r-project.org/) was used for all analyses. $P$ values, if reported, are two sided.

\section{Sample size justification}

Power for the Wilcoxon-Mann-Whitney-derived non-inferiority testing was based on 10000 simulated trials. We simulated pain scores (during the recovery room) from a discrete distribution with median (IQR) $2(0-3) .{ }^{20}$ Between the quartiles, the probability of each score was assumed constant. The distribution for each group was assumed to be the same (ie, equivalence). The sample size of 50 per group provided $81 \%$ power to detect noninferiority in pain (online supplemental appendix 1). Similarly, opioid consumption (combined operating and recovery room amounts) was assumed to follow a truncated normal distribution with mean $2.5 \mathrm{mg}$ and SD $2 \mathrm{mg}$, and minimum value $0 \mathrm{mg}$. The sample size of 50 per group provided at least $95 \%$ power to detect non-inferiority with margin $2 \mathrm{mg}$ (online supplemental appendix 2). Therefore, the planned enrolment for this study was 50 subjects for each of the two treatment groups with a total enrolment of 100 subjects.

\section{RESULTS}

One hundred subjects were enrolled in the 21 months beginning May 2019 (figure 1). All subjects were randomized to either serratus block $(n=49)$ or PVB $(n=51)$ treatment groups after confirmation of adequate ultrasound visualization, and all interventions were performed per protocol. All subjects completed the outcome measurements and follow-up call or visit on postoperative day (POD) 1. There were no postrandomization exclusions of any subjects due to protocol deviations.

Anthropometric and surgical characteristics were well balanced between the two treatment groups (tables 1 and 2).

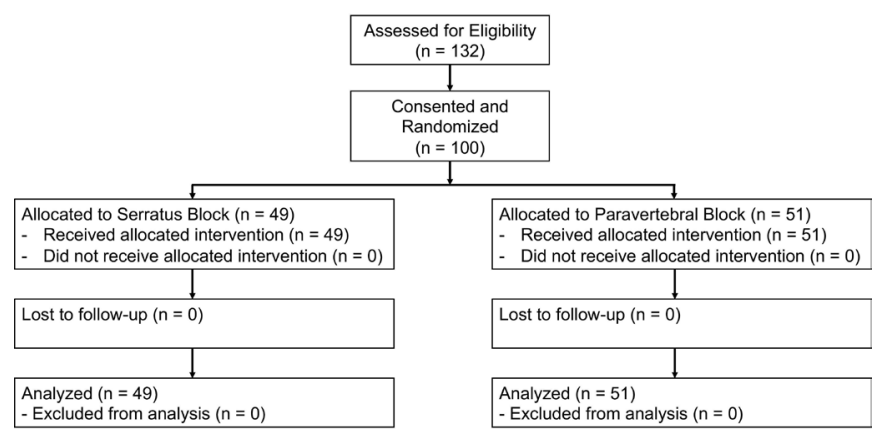

Figure 1 Consolidated standards of reporting trials statement diagram showing flow of study participants.

Compared with PVBs, the median time to perform the serratus block procedure as measured from time of block needle insertion to removal of block needle was shorter to a statistically significant degree $(1.7 \mathrm{~min} / \mathrm{side}$ for serratus blocks vs $4.3 \mathrm{~min} / \mathrm{side}$ for PVBs; $\mathrm{p}<0.001$ ) (table 3$)$. The median average NRS pain scores during the block procedures were similar (1.5 for serratus block vs 1.6 for PVBs; $\mathrm{p}=0.94$; table 3 ), as were the median maximum NRS pain scores (2.4 for serratus block vs 2.7 for PVB; $p=0.85$; table 3).

\section{Primary end point}

Within the recovery room, pain scores for participants with serratus blocks $(n=49)$ was a median (IQR) of $4.0(0-5.5)$ vs $0(0-3.0)$ for those with PVBss $(n=51)$ : $95 \%$ CI -3.00 to $-0.00 ; p=0.001$ (figure 2). The lower limit of the $95 \%$ CI was less than -1.25 , which is lower than the prespecified framework for non-inferiority, and therefore, non-inferiority was not demonstrated. Note that the upper limit is less than 0-5 decimal places $\left(-2.66 \times 10^{-5}\right)$, demonstrating inferiority. However, the difference in MEQ did not reach statistical significance for paravertebral superiority with the serratus group consuming $14 \mathrm{mg}$ (10-19) vs $10 \mathrm{mg}(10-16)$ for the paravertebral group: 95\% CI -4.50 to $0.00, p=0.12$ (figure 3 ). In this case, the upper limit of the $95 \%$ CI was on the positive side of 0-6 decimal places $\left(2.49 \times 10^{-6}\right)$. Since the $95 \%$ CI lower limit of -4.5 was less than our prespecified margin of -2.0 , we failed to conclude that the serratus block is non-inferior to the PVB.

\section{Secondary end points}

There was minimal difference in median operating room opioid administration between the two treatment groups $(10.0 \mathrm{mg}$ MEQ for serratus block group vs $10.0 \mathrm{mg}$ for the PVB group; $\mathrm{p}=0.38$; table 4). However, there was increased opioid use in the PACU in the serratus block versus PVB cohort $(4.5 \mathrm{mg} \mathrm{v} 0 \mathrm{mg}$,

Table 1 Anthropometric and surgical characteristics

\begin{tabular}{llll}
\hline Factor & Serratus block $(\mathrm{n}=49)$ & $\begin{array}{l}\text { Paravertebral block } \\
(\mathrm{n}=51)\end{array}$ & $\begin{array}{l}\text { Standardized } \\
\text { difference }\end{array}$ \\
\hline Age (years) & $55.0(44.0-64.0)$ & $53.0(41.5-61.5)$ & 0.21 \\
Height $(\mathrm{cm})$ & $163.0(158.0-169.0)$ & $164(159.0-170.0)$ & 0.33 \\
Weight $(\mathrm{kg})$ & $68.2(61.4-79.5)$ & $73.6(61.8-85.7)$ & 0.19 \\
Female sex & $49(100 \%)$ & $48(94.1 \%)$ & 0.35 \\
$\begin{array}{l}\text { Bilateral } \\
\text { procedure }\end{array}$ & $17(34.7 \%)$ & $19(38.0 \%)$ & 0.22 \\
\hline
\end{tabular}

Data are presented as median (IQR) or number (percentage).

Test used: Cohen's d was used to calculate the standardized difference between treatment groups. Differences larger than 0.392 were considered imbalanced. 


\begin{tabular}{|c|c|c|}
\hline Factor & $\begin{array}{l}\text { Serratus block } \\
(n=49),(\%)\end{array}$ & $\begin{array}{l}\text { Paravertebral block } \\
(n=51),(\%)\end{array}$ \\
\hline $\begin{array}{l}\text { Lumpectomy with axillary lymph } \\
\text { node biopsy }\end{array}$ & $32(65)$ & $29(57)$ \\
\hline $\begin{array}{l}\text { Lumpectomy with axillary lymph } \\
\text { node dissection }\end{array}$ & $3(6)$ & $2(4)$ \\
\hline Breast reconstruction & $12(25)$ & $12(24)$ \\
\hline Breast reduction & $2(4)$ & $4(8)$ \\
\hline Breast implant insertion/exchange & $11(22)$ & $8(16)$ \\
\hline
\end{tabular}

Some patients underwent multiple procedures during their surgery.

respectively; $\mathrm{p}=0.03$ ). The highest reported NRS pain scores in the PACU were $5.0 \mathrm{v} 4.0$ in the serratus and PVB cohorts, respectively $(\mathrm{p}=0.04)$. There was a difference in anti-emetic use between the two treatment groups, in which 19 (39\%) in the serratus block and $8(16 \%)$ in the PVB group required antiemetics in the PACU $(\mathrm{p}=0.01)$ (table 4$)$.

Eleven subjects were admitted postoperatively, in which 5 $(10 \%)$ and $6(12 \%)$ were in the serratus block and PVB cohort, respectively. Of note, inadequate pain control was not the reason for admission for any of the subjects. Rather, the reason for admission were due to surgical reasons and need for overnight monitoring. All hospitalized subjects were discharged on POD 1. The number of hospitalized subjects was too small to make any conclusions regarding differences between groups in day of discharge.

On the morning following surgery, median NRS pain scores were higher in the serratus block versus $\mathrm{PVB}$ cohort for highest score $(5.0 \vee 3.0, \mathrm{p}<0.01)$, lowest score $(1.0 \vee 0, \mathrm{p}=0.001)$, and average score $(3.0 \mathrm{v} 2.0, \mathrm{p}=0.01)$ (table 4$)$. There was little difference in opioid use on POD1 between serratus block and PVB groups ( $2 \mathrm{mg}$ v $2 \mathrm{mg}, \mathrm{p}=0.24)$. No differences were also seen between median nausea Likert scores and number of overnight awakenings due to pain (table 4). There were no protocol deviations or adverse events related to the block procedures.

\section{DISCUSSION}

In our study, serratus blocks provided inferior analgesia compared with PVBs; and, regarding opioid requirements, we were unable to demonstrate at least non-inferiority of serratus blocks. Furthermore, there were several differences in our secondary outcomes favoring PVB, including need of antiemetics in the PACU, opioid consumption in the PACU, and worst/least NRS pain scores in the PACU and up to POD 1. Of note, all blocks were performed by fellowship-trained attendings or current fellows overseen by the attendings who all have a great deal of experience with both blocks. Thus, providers with less experience-especially with PVBs-may still elect to perform

\begin{tabular}{llll}
\hline Table 3 & \multicolumn{1}{l}{ Block procedure data } & & \\
\hline Factor & $\begin{array}{l}\text { Serratus block } \\
(\mathbf{n}=49)\end{array}$ & $\begin{array}{l}\text { Paravertebral } \\
\text { block }(\mathbf{n}=51)\end{array}$ & P value \\
\hline $\begin{array}{l}\text { Time to perform procedure per } \\
\text { side (min) }\end{array}$ & $1.25(0-4.0)$ & $4.0(2.0-6.0)$ & $<0.001$ \\
$\begin{array}{l}\text { Average pain during block } \\
\text { placement (NRS) }\end{array}$ & $1.0(0-2.0)$ & $1.0(0-2.0)$ & 0.94 \\
\hline $\begin{array}{l}\text { Worst pain during block (NRS) } \\
2.0(0-4.0)\end{array}$ & $2.0(1.0-3.5)$ & 0.85 \\
\hline
\end{tabular}

Data are presented as median (IQR).

Test used: Wilcoxon-Mann-Whitney test. NRS, Numeric Rating Scale.

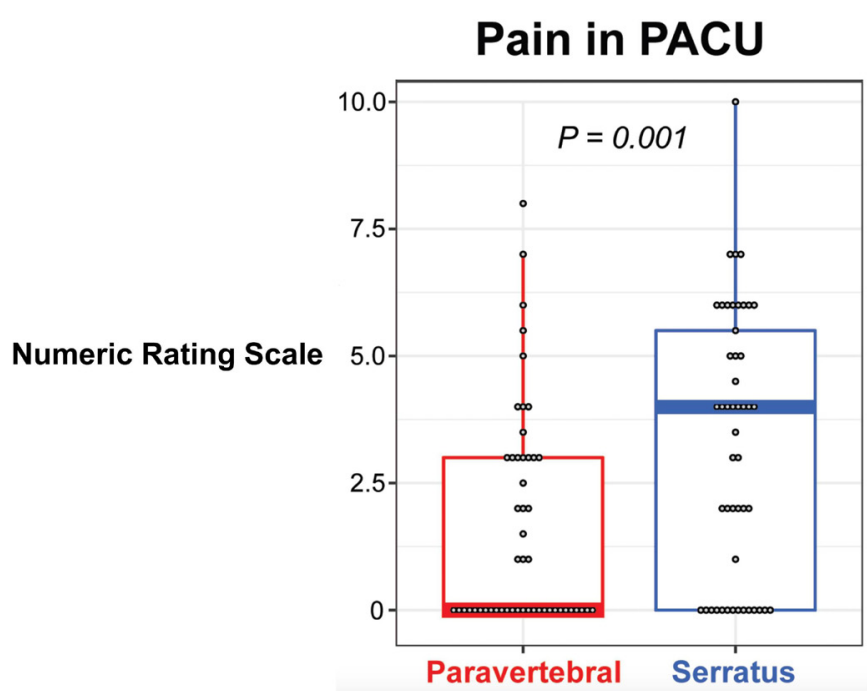

Figure 2 Median Numeric Rating Scale pain scores in postanesthesia care unit (PACU) between two treatment groups. Horizontal lines indicate overall median for each treatment group; boxes indicate the 25th and 75th percentiles; vertical lines indicate the 5thand 95th percentiles. Each dot represents a subject's score.

the less efficacious fascial plane block due to risk/benefit ratio concerns; while practitioners skilled at PVB have reason not to change their current practice for breast surgery.

In one study, continuous serratus anterior plane catheters were compared with surgically placed continuous PVB catheters for patients undergoing videoscopic-assisted thoracic surgery in a randomized controlled trial. ${ }^{21}$ Serratus blocks were shown to be non-inferior compared with surgically placed PVB in their primary outcome of 48-hour opioid consumption. In another randomized trial, surgically placed serratus anterior plane catheters were compared with ultrasound-guided catheter placements of PVB in patients undergoing thoracotomy, ${ }^{22}$ in which

\section{Opioid Consumption}

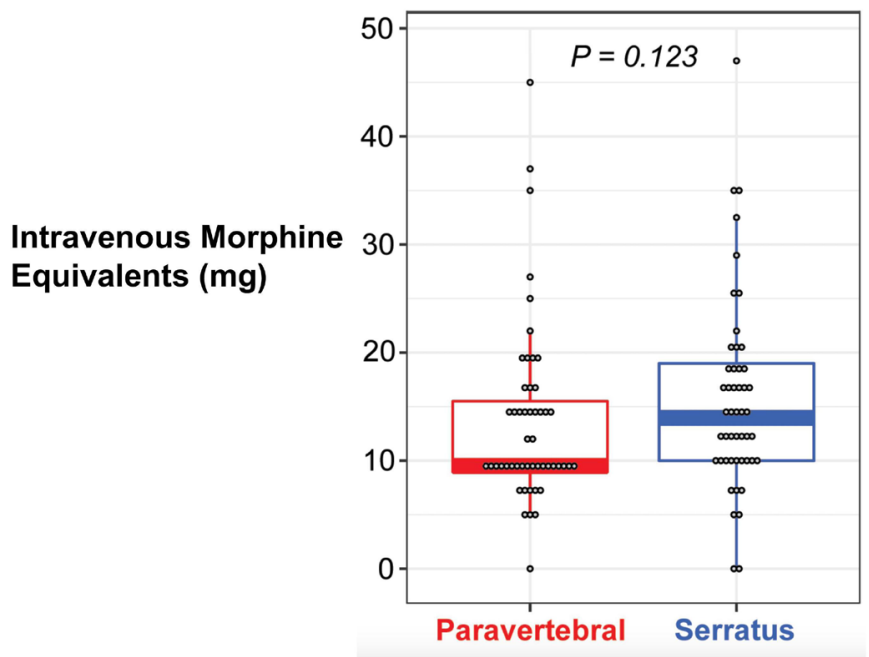

Figure 3 Operating room and postanesthesia care unit intravenous morphine equivalents opioid consumption between two treatment groups. Horizontal lines indicate overall median for each treatment group; boxes indicate the 25th and 75th percentiles; vertical lines indicate the 5 th and $95^{\text {th }}$ percentiles. Each dot represents a subject's opioid use. 


\begin{tabular}{|c|c|c|c|}
\hline Factor & $\begin{array}{l}\text { Serratus block } \\
(n=49)\end{array}$ & $\begin{array}{l}\text { Paravertebral } \\
\text { block }(n=51)\end{array}$ & $P$ value \\
\hline \multicolumn{4}{|l|}{ Median MEQs in mg (IQR) } \\
\hline Operating room & $10.0(10.0-10.0)$ & $10(8.75-10.0)$ & 0.38 \\
\hline PACU & $4.5(2.0-7.0)$ & $0(0-6.5)$ & 0.03 \\
\hline $\begin{array}{l}\text { PACU discharge to } \\
\text { morning of POD1 }\end{array}$ & $2.0(2.0-4.0)$ & $2.0(0-4.0)$ & 0.24 \\
\hline $\begin{array}{l}\text { Antiemetics given in the } \\
\text { PACU (\%) }\end{array}$ & $19(38.8 \%)$ & $8(15.7 \%)$ & 0.01 \\
\hline \multicolumn{4}{|c|}{ Median pain NRS scores in PACU (IQR) } \\
\hline Highest & $5.0(4.0-7.0)$ & $4.0(0-6.5)$ & 0.04 \\
\hline Lowest & $0(0-3.0)$ & $0(0-0)$ & 0.01 \\
\hline \multicolumn{4}{|c|}{ Median pain NRS scores from PACU discharge to morning of POD1 in mg (IQR) } \\
\hline Highest & $5.0(3.0-6.0)$ & $3.0(2.0-5.0)$ & $<0.01$ \\
\hline Lowest & $1.0(0-3.0)$ & $0(0-1.0)$ & $<0.01$ \\
\hline Average & $3.0(2.0-4.0)$ & $2.0(0.5-3.0)$ & 0.01 \\
\hline $\begin{array}{l}\text { Median Likert scale } \\
\text { nausea morning of POD1 } \\
\text { (IQR) }\end{array}$ & $0(0-0)$ & $0(0-0)$ & 0.63 \\
\hline $\begin{array}{l}\text { Median no of awakenings } \\
\text { due to pain (IQR) }\end{array}$ & $0(0-0)$ & $0(0-0)$ & 0.31 \\
\hline
\end{tabular}

Data are presented as median (IQR).

Test used:Wilcoxon-Mann-Whitney test (continuous factors), Fischer's exact test (categorical). Likert scale: $0=$ nausea; $10=$ vomiting.

NRS, Numeric Rating Scale; PACU, postanesthesia care unit; POD, postoperative day.

no difference was found in any of the pain outcomes. Our study differs from these as both serratus blocks and PVB were placed by expert regional anesthesiologist via ultrasound-guidance, were single injections, and a different surgical population.

In a randomized controlled three-arm study comparing PVB, serratus blocks, and PECS-2 block for breast surgery, investigators found that there was no difference in PVB vs serratus block in terms of postoperative opioid requirements. ${ }^{14}$ However, in this study, only 15 subjects were included in each cohort and thus may have been underpowered. Furthermore, PVB blocks were performed at only one level compared with the two levels performed in our study. After recruiting 100 subjects, we were able to show a statistically significant difference in postoperative pain scores. Furthermore, a randomized trial comparing sham block to serratus anterior plane block in patients undergoing simple or partial mastectomy demonstrated that there was no difference in quality of recovery-15 scores and analgesia outcomes between both cohorts. ${ }^{23}$ Their results are in line with ours as we were unable to claim non-inferiority of serratus blocks compared with PVBs.

While the mechanism of analgesia with PVB has been discerned-direct blockade of spinal nerves in the paravertebral space-it is unclear the reliability of nerve blockade when local anesthetic is deposited between targeted fascial planes. For example, it is theorized that with erector spinae plane blocks, local anesthetic eventually diffuses across the intercostal membrane and into the paravertebral space-however; the reliability of providing subsequent analgesia is in question. In a recent randomized controlled trial, PVBs were superior to erector spinae plane blocks for both postoperative opioid consumption and pain scores. ${ }^{24}$ The study demonstrated that the efficacy of erector spinae plane block-while theoretically technically easier to perform-is not as effective for breast surgery analgesia as PVB.

Given our findings, we can only speculate as to why PVB provided superior analgesia in terms of postoperative pain scores compared with serratus blocks. Both blocks aim to target similar nerves that innervate the chest wall (ie, thoracic intercostal nerves). With PVB, the nerve roots are more reliability targeted and thus subsequent blockade of the targeted intercostal nerves are blocked with local anesthetic. Therefore, unlike serratus blocks, PVBs blocks all the branches of the intercostal nerve-posterior, lateral, and anterior. This is important because the dermatomal distribution of these branches may vary across patients. The serratus block relies on deposition of local anesthetic to spread and block intercostal nerves traveling in that fascial plane-not all nerves may be adequately blocked in this case. ${ }^{25}$ Thus, as only the lateral branches are targeted in serratus blocks, this may result in incomplete coverage of the surgical area. In a cadaveric study where dye was injected via a serratus block approach, the lateral cutaneous branches of the intercostal nerves were consistently stained while the intercostal nerve itself was not stained each time. ${ }^{12}$ This suggests that serratus blocks may be reasonably adequate to provide analgesia in the superficial regions of the lateral thorax, but not always the case when deeper structures are involved. Furthermore, there is variability in cephalad spread of local anesthetic within the plane following a serratus block. ${ }^{26}$

\section{Limitations}

Although the subjects of this investigation were blinded to treatment group assignment, investigators were aware of the randomization results to enable block administration. However, data for the dual primary outcome measures were collected by healthcare providers blinded to treatment group allocation. Furthermore, it was difficult to completely blind subjects as the positioning of each block differed (ie, lateral for serratus block and sitting for PVB); however, patients received sedation prior to positioning. In addition, the results apply only to the specific local anesthetic type, concentration and volume of the current study. Similarly, different surgical procedures (eg, mastectomy), block techniques (eg, in vs out of plane), and administration approaches (eg, lossof-resistance vs ultrasound) would probably alter the results. Our study population was not completely homogenous, and included a variety of non-mastectomy surgeries.

More specifically, a potential confounder of the results could be that serratus block subjects received one injection whereas PVB subjects received two injections. Although our goal was to compare our institutional practice of two-level PVBs to the customary single-level serratus block-increasing external validity-this difference may have impacted the blinding of subjects as well as the analgesic results. In addition, intraoperative opioid administration by the blinded operating room anesthesia provider was not standardized and was left to their discretion.

Another limitation of our study included the time interval of pain assessments in our primary outcome. Since pain was only recorded in the PACU, analgesia was not fully assessed for the duration of the blocks in both treatment groups. Since most of these surgeries were performed on an outpatient basis, we chose to limit our blinded pain assessments to the PACU. Furthermore, despite differences in NRS pain scores, we did not find a difference opioid consumption; which may have required a higher-powered study for that outcome. Nonetheless, we are also unable to claim at least non-inferiority in regard to opioid consumption. Lastly, since mastectomy subjects were excluded from our study, the results only apply to non-mastectomy breast surgery. 


\section{CONCLUSIONS}

This randomized active controlled study suggests that PVB provides improvements in short-term postoperative analgesia compared with serratus blocks following non-mastectomy breast surgery.

Contributors RG, MCD and BMI: this author helped with literature search, data collection, study design, analysis of data, manuscript preparation and review of manuscript; MWS, AA, SB, MD, AS and CR: this author helped with literature search, study design, manuscript preparation and review of manuscript; JFS, BPC, ETS, WBA, BK, BSA, JJF and AMW: this author helped with literature search, data collection, study design, manuscript preparation and review of manuscript.

Funding The authors have not declared a specific grant for this research from any funding agency in the public, commercial or not-for-profit sectors.

Competing interests The University of California has received funding and product for other research projects from Epimed International (Farmers Branch, Texas, USA); Infutronics (Natick, Massachusetts, USA); and SPR Therapeutics (Cleveland, Ohio, USA) for the following authors: RG, MWS, JFS, ETS, BK, JJF, AMW and BMI. RG is a consultant for Avanos (Alpharetta, Georgia, USA).

Patient consent for publication Not required.

Ethics approval UCSD Human Research Protections Program (HRPP).

Provenance and peer review Not commissioned; externally peer reviewed.

Data availability statement Data are available on reasonable request. Data contains PHI and is institutional data. Request for data would have to abide by HIPAA compliance rules.

Open access This is an open access article distributed in accordance with the Creative Commons Attribution Non Commercial (CC BY-NC 4.0) license, which permits others to distribute, remix, adapt, build upon this work non-commercially, and license their derivative works on different terms, provided the original work is properly cited, appropriate credit is given, any changes made indicated, and the use is non-commercial. See: http://creativecommons.org/licenses/by-nc/4.0/.

ORCID iDs

Engy T Said http://orcid.org/0000-0002-7897-1670

Brenton S Alexander http://orcid.org/0000-0003-3657-0673

\section{REFERENCES}

1 Fecho K, Miller NR, Merritt SA, et al. Acute and persistent postoperative pain after breast surgery. Pain Med 2009;10:708-15.

2 Habib AS, Kertai MD, Cooter M, et al. Risk factors for severe acute pain and persistent pain after surgery for breast cancer: a prospective observational study. Reg Anesth Pain Med 2019;44:192-9.

3 Ilfeld BM, Madison SJ, Suresh PJ, et al. Persistent postmastectomy pain and pain-related physical and emotional functioning with and without a continuous paravertebral nerve block: a prospective 1-year follow-up assessment of a randomized, triple-masked, placebo-controlled study. Ann Surg Oncol 2015;22:2017-25

4 Macdonald L, Bruce J, Scott NW, et al. Long-Term follow-up of breast cancer survivors with post-mastectomy pain syndrome. Br J Cancer 2005;92:225-30.

5 Cheng GS, Ilfeld BM. An evidence-based review of the efficacy of perioperative analgesic techniques for breast cancer-related surgery. Pain Med 2017;18:1344-65.

6 Schnabel A, Reichl SU, Kranke P, et al. Efficacy and safety of paravertebral blocks in breast surgery: a meta-analysis of randomized controlled trials. $\mathrm{Br} J$ Anaesth 2010;105:842-52.
7 Greengrass R, O'Brien F, Lyerly K, et al. Paravertebral block for breast cancer surgery. Can J Anaesth 1996;43:858-61.

8 Pace MM, Sharma B, Anderson-Dam J, et al. Ultrasound-Guided thoracic paravertebral blockade: a retrospective study of the incidence of complications. Anesth Analg 2016;122:1186-91.

9 Forero M, Adhikary SD, Lopez H, et al. The erector spinae plane block: a nove analgesic technique in thoracic neuropathic pain. Reg Anesth Pain Med 2016:41:621-7.

10 Blanco R, Fajardo M, Parras Maldonado T. Ultrasound description of PecS II (modified PecS I): a novel approach to breast surgery. Rev Esp Anestesiol Reanim 2012;59:470-5.

11 Blanco R, Parras T, McDonnell JG, et al. Serratus plane block: a novel ultrasoundguided thoracic wall nerve block. Anaesthesia 2013;68:1107-13.

12 Mayes J, Davison E, Panahi P, et al. An anatomical evaluation of the serratus anterior plane block. Anaesthesia 2016;71:1064-9.

13 Desai M, Narayanan MK, Venkataraju A. Pneumothorax following serratus anterior plane block. Anaesth Rep 2020;8:14-16.

14 Jain D, Mohan VK, Bhoi D, et al. Analgesic efficacy and spread of local anesthetic in ultrasound-guided paravertebral, pectoralis II, and serratus anterior plane block for breast surgeries: a randomized controlled trial. Saudi J Anaesth 2020;14:464-72.

15 Gupta K, Srikanth K, Girdhar KK, et al. Analgesic efficacy of ultrasound-guided paravertebral block versus serratus plane block for modified radical mastectomy: a randomised, controlled trial. Indian J Anaesth 2017;61:381-6.

16 Mahran E, Adlan S, Alieldeen N. Comparative randomized study of continuous serratus anterior plane block versus continuous paravertebral block in post-mastectomy pain. Ain-Shams Journal of Anesthesiology 2020;12.

17 Saad FS, El Baradie SY, Abdel Aliem MAW, et al. Ultrasound-Guided serratus anterior plane block versus thoracic paravertebral block for perioperative analgesia in thoracotomy. Saudi J Anaesth 2018;12:565-70.

18 Huang $L$, Zheng $L$, Wu B, et al. Effects of ropivacaine concentration on analgesia after ultrasound-guided serratus anterior plane block: a randomized double-blind trial. J Pain Res 2020;13:57-64.

19 Schumi J, Wittes JT. Through the looking glass: understanding non-inferiority. Trials 2011;12:106.

20 Ilfeld BM, Madison SJ, Suresh PJ, et al. Treatment of postmastectomy pain with ambulatory continuous paravertebral nerve blocks: a randomized, triple-masked, placebo-controlled study. Reg Anesth Pain Med 2014;39:89-96.

21 Hanley C, Wall T, Bukowska I, et al. Ultrasound-Guided continuous deep serratus anterior plane block versus continuous thoracic paravertebral block for perioperative analgesia in videoscopic-assisted thoracic surgery. Eur J Pain 2020;24:828-38.

22 Patel A, Kumar V, Garg R, et al. Comparison of analgesic efficacy of ultrasound-guided thoracic paravertebral block versus surgeon-guided serratus anterior plane block for acute postoperative pain in patients undergoing thoracotomy for lung surgery-A prospective randomized study. Saudi J Anaesth 2020;14:423-30.

23 Abdallah FW, Patel V, Madjdpour C, et al. Quality of recovery scores in deep serratus anterior plane block vs. sham block in ambulatory breast cancer surgery: a randomised controlled trial. Anaesthesia 2021. doi:10.1111/anae.15373. [Epub ahead of print: 25 Jan 2021]

24 Swisher MW, Wallace AM, Sztain JF, et al. Erector spinae plane versus paravertebral nerve blocks for postoperative analgesia after breast surgery: a randomized clinical trial. Reg Anesth Pain Med 2020;45:260-6.

25 Biswas A, Castanov V, Li Z, et al. Serratus plane block: a cadaveric study to evaluate optimal injectate spread. Reg Anesth Pain Med 2018;43:854-8.

26 Daga V, Narayanan MK, Dedhia JD, et al. Cadaveric feasibility study on the use of ultrasound contrast to assess spread of injectate in the serratus anterior muscle plane Saudi J Anaesth 2016;10:198-201. 